\title{
Lifetime Test of Tracked Vehicle Torsion Bars using Monte Carlo Method
}

\author{
Jan Furch ${ }^{a^{*}}$, Quy Hung Nguyen ${ }^{\mathrm{b}}$ \\ ${ }^{a}$ University of Defence, Kounicova 65, Brno, 66210, Czech Republic \\ ${ }^{b}$ Institute of Mechanical Engineering, Dong Quan 42, Hanoi, 122100, Vietnam
}

\begin{abstract}
The article deals with the service life of torsion bars in tracked vehicles. The aim of the article is to show the suitability of accelerated tests and modelling for determining the service life of torsion bars, which takes many years in real operation. The design of a test bench for accelerated tests is presented together with limiting conditions, which were afterwards verified. Subsequently, a virtual model of the torsion bar of a tracked vehicle was created with the help of finite elements. Dynamic modelling was performed by MSC Adams software with a module using finite elements. Furthermore, the article shows the possibility of using the Monte Carlo method to determine the service life of torsion bars of tracked vehicles. The Monte Carlo utility of Accelerated Life Testing Analysis (ALTA) software is used to obtain failure data at specified test stress levels. Using the Monte Carlo simulation, one data set is generated containing values that are arranged in dependence on the specific lifetime distribution of the Weibull distribution. Finally, a comparison of the experiment with the calculated values is performed. The results obtained can be used to compile an accelerated test plan. This modelling design saves a lot of money and time to determine the life of the torsion bar in tracked vehicles.
\end{abstract}

\section{Keywords:}

Suspension;

Torsion Bar;

Test Rig;

FE Part;

Lifetime Test;

Accelerated Life Testing;

Monte Carlo Method.

\section{Article History:}

$\begin{array}{llll}\text { Received: } & 15 & \text { June } & 2020 \\ \text { Accepted: } & 19 & \text { September } & 2020 \\ \text { Published: } & 01 & \text { October } & 2020\end{array}$

\section{1- Introduction}

The combat vehicle is constructed for advance movement especially in a heavy terrain. The transmission mechanical system has to work under the heavily dynamically stress while the overcoming of heavy terrain (mud, snow, obstacles etc.). It is required that the combat vehicle has to work with the high performance and reliable transmission [1]. High speed tracked vehicles are usually fitted with a suspension system employing torsion bars and shock absorbers to mitigate the terrain-induced shocks and attenuate the vibrations of the vehicle body. The torsion bar substitutes the coil spring in earlier tracked vehicles as it performs spring actions with improved characteristics [2]. In order to evaluate the performance and ride comfort of the vehicle, it is necessary to investigate characteristics of the suspension spring element, whose parameter is the torsion bar stiffness. Lifetime calculations for machine components represent an important foundation for quantitative reliability methods. For a prediction of a lifetime, all failure causes must be known. These can be divided into three categories [3, 4]:

- Fatigue failures, aging failures, wear out failures and failures caused by environmental influences, for example corrosion, etc., caused by changes in the used materials, dependent upon time, e.g. highly loaded components in automotive technology;

- Tolerance failures lead to unreliable deviations, which forbid an efficient function, for example machine tools, which no longer achieve desired production precision, or seals, which show an unreliable high leakage;

\footnotetext{
*CONTACT: Jan.furch@unob.cz

DOI: http://dx.doi.org/10.28991/esj-2020-01238
}

(C) 2020 by the authors. Licensee ESJ, Italy. This is an open access article under the terms and conditions of the Creative Commons Attribution (CC-BY) license (https://creativecommons.org/licenses/by/4.0/). 
- Failures caused by faults, which occur during production, assembly or during the operation of machines.

To obtain characteristics of the torsional bar, a test rig with a measuring system was designed and constructed to measure the twisting moment acting on torsion bar and the corresponding twist angle. The test rig provides a sinusoidal excitation to the torsion bar with the same working conditions as the vehicle on road. After that, the design and simulation of the test rig were carried out by the aid of MSC Adams software.

When test acceleration is accomplished with a single stress only, the models are life stress models, where the damage per unit time of test is appropriately accelerated by increasing the level of stress.

The three most frequently used relationships are [4]:

a) inverse power law model, used for test acceleration when stresses other than constant temperature are considered, such as electrical, mechanical, chemical (corrosion) and others;

b) Arrhenius reaction rate model, used for constant temperature stresses, based on the effect that the absolute temperature has on a failure mechanism;

c) Eyring model which is used in cases where the acceleration is achieved with temperature and moisture stress levels. The model is derived from quantum mechanics.

With all acceleration models, test data can be analyzed using established analytical models to determine characteristic accelerated life parameters. Using the acceleration factors, the parameters corresponding to use environments are determined and used for reliability projections as needed. The acceleration models should if possible be verified by plotting the test data.

\section{2- Planning Lifetime Test}

Planning lifetime tests can be divided into experimental-technical measurement planning and statistical test planning [4].

\section{2-1- Experimental-technical Measurement Planning}

The common fundamental principles for correct execution of an experiment apply. The most important of these principles are as listed, the inverse power law is applicable to:

- The boundary conditions and limits must be exactly defined and kept.

- For lifetime tests this is especially important for the load spectrum.

- The technical measurement process for the registration and control of the boundary conditions must be established along with their accuracy. Depending upon the resources, more information is acquired at the test stand than actually needed.

- If longer testing times are expected, then the use of automated and/or computer controlled measured value gathering and control equipment should be strived for.

- For a determination of the lifetime, the exact specification of a limit value is necessary, at which the nominal function is no longer fulfilled. If the damage is a continuously changing value, as for example a leak volume for a seal.

- The control equipment must be built up in such a way that the primary failure cause can even be determined after the failure effect. This is important since each failure mode is assigned its own characteristic reliability parameters [5].

\section{2-2- Statistical Test Planning}

Accelerated life tests are component life tests with components operated at high stresses and failure data observed. Test planning means picking stress levels, sample sizes and test times to produce enough data to fit models and make projections. It is good design practice to put more test units in the lower stress cells, to make up for the fact that these cells will have a smaller proportion of units failing.

In statistical test planning, the first step involves determining the size of the inspection lot. The inspection lot size is in close connection with the confidence levels and the statistical spread of the measured values. If fewer components are tested, then the result of the statistical assessment becomes more uncertain. For an accurate result, it is necessary that a sufficient quantity of components is tested. This can increase the time and effort involved in a test immensely. Figure 1 shows the influence of number of test units on the reliability. It is obvious that with the diminishing of the sample size, the reliability is decreasing. 


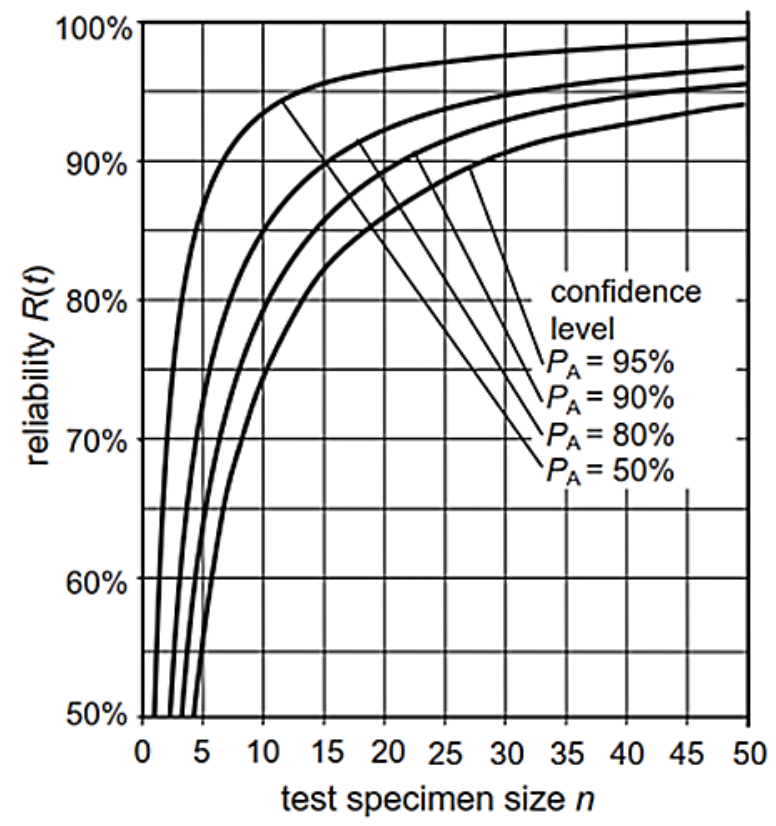

Figure 1. Minimal reliability $R(t)$ as a function of the test specimen size $n$ and the confidence level $P_{A}$, if at the point in time $t$ no failure has occurred [5].

Another important point in statistical test planning involves establishing a suitable test strategy. Possible strategies include:

a) Complete tests - The best statistical option is a complete test, in which all components of a test specimen are subjected to a lifetime test. This means that the test is run until the last element has failed. Thus, failure times for all elements are available for further assessment.

b) Incomplete (censored) tests - In order to reduce the time and effort involved in a test stand, it is reasonable to carry out incomplete tests, also known as censored tests. Here the test is carried out until a certainly predetermined lifetime or until a certain number of failed components has been reached. Such tests are not as meaningful as complete tests but are often connected with a considerably lower time and effort at the test stand.

c) Strategies for shortening test times.

Another option for a considerably shorter test time is the Sudden Death Test and tests with an increased load. There are different types of accelerated life test (ALT) plans in use, which include subjective, traditional, best traditional, statistically optimum and compromise plans [6-8]. As with the simpler ALTs, evaluation of test plan properties can be done using either large-sample approximations or simulation methods [6].

Monte Carlo simulation provides a powerful, insightful tool for planning experiments. For a specified model and planning values for the model parameters, it is possible to use a computer to simulate ALT experiments to see the kind of data that will be obtained and to visualize the variability from trial to trial. Such simulations provide an assessment of sampling uncertainty that will result from using a limited number of test specimens [9].

\section{3- Theory Planning Accelerated Life Test}

Before starting an accelerated life test, it is advisable to have a plan that helps in accurately estimating reliability at operating conditions while minimizing test time and costs. To design the plan for the accelerated life tests it is necessary to establish the selected parameters:

a) The acceleration model. For accelerated life tests where the failure mechanism is a mechanical one (the fatigue failure), the most adequate one is the IPL model. The inverse power law (IPL) model is commonly used for nonthermal accelerated stresses. This model was calculated by the Equations 1 to 3 . With the inverse power law, the characteristic that represents product reliability related to time, such as characteristic life, mean life, mean time to a failure, is represented as [4, 22-24]:

$L(S)=C^{-1} \times S^{-m}$

Where: $L(S)=$ is the life or other predetermined time duration as a function of stress; $C$ is one of the model parameters $(C>0)$ to be determined; $S$ represents the stress level; $m$ is another model parameter, dependent on stress behavior, also to be determined. 
The power law model is simple when expressed or plotted in logarithmic form, where it becomes a straight line with the slope representing the value of parameter $m$, and the value of the intercept with the $y$-axis is a function of the constant $S$ [4]:

$\ln [L(\mathrm{~S})]=-m \ln (S)-\ln (C)$

The inverse power law is applicable to all distributions regularly used in reliability. The test acceleration factor is then [4]:

$A_{S_{-} I P L}=\frac{L\left(S_{U s e)}\right.}{L\left(S_{T e s t}\right)}=\frac{C^{-1} \times S_{U s e}^{-m}}{C^{-1} \times S_{T e s t}^{-m}}=\left(\frac{S_{T e s t}}{S_{U s e}}\right)^{m}$

Where: $A_{S-I P L}$ is the acceleration factor of stress by inverse power law; $L\left(S_{U s e}\right)$ is the life as a function of stress in actual use; $L\left(S_{\text {Test }}\right)$ is the as a function of stress applied in test.

Parameter $C$ in the test acceleration cancels out, but the parameter $m$ shall be determined for the item and the stress type. The parameter $m$ in the inverse power relationship is a measure of the effect of the stress on the life. As the absolute value of $m$ increases, the greater is the effect of the stress. Negative values of $m$ indicate an increasing life with increasing stress. An absolute value of $m$ approaching zero indicates small effect of the stress on the life, with no effect (constant life with stress) when $m=0$.

a) The available test time: Choose the duration of the accelerated life test 360000 cycles.

b) The number of units subjected to accelerated life tests: suppose 20 specimens.

c) The distribution law of the number of cycles until failure used in accelerated life testing: choose Weibull distribution and give shape parameter.

d) The stress under normal use condition and maximum in accelerated condition: the twist angle in normal condition is $30^{\circ}$ and the maximum angle is $50^{\circ}$ (during the experiment only $40^{\circ}$ ).

e) The accelerated life test plan: choose three levels best compromise plan. This plan recommends three stress levels: a high stress level, which is the maximum allowable stress value that you specified during setup, a low stress level and a middle stress level.

f) The test plan was realized using the ALTA software, introducing the aforementioned parameters. The recommended test plan suggested stress levels to be used in the test and the recommended allocation of units to each stress level.

Table 1. Recommended test plan [4].

\begin{tabular}{ccccc}
\hline Stress level & $\begin{array}{c}\text { Stress value } \\
\left({ }^{\circ}\right)\end{array}$ & $\begin{array}{c}\text { Unit allocation } \\
(\boldsymbol{\%})\end{array}$ & $\begin{array}{c}\text { Unit allocation } \\
\left(\mathbf{Q}_{\mathrm{ty}}\right)\end{array}$ & $\begin{array}{c}\text { Probability of failure } \\
(-)\end{array}$ \\
\hline Low stress level & 40 & 47.3 & 9.46 & 0.211 \\
Middle stress level & 45 & 25 & 5 & 0.648 \\
High stress level & 50 & 27.7 & 5.54 & 0.99 \\
\hline
\end{tabular}

The plan recommended 3 stress levels at $40^{\circ}, 45^{\circ}, 50^{\circ}$ and number of units for each stress level. That was 9 units to test at twist angle $40^{\circ}, 5$ units at $45^{\circ}$ and 6 units at $50^{\circ}$.

\section{4- Experiment Description}

\section{4-1- Characteristics of Torsion Bar}

This chapter describes the practical implementation of the accelerated life test for the torsion bar of the combat track vehicle. The object of the test was a torsion bar of the track vehicle (thereafter called torsion bar). The section portrays the experimental test rig for imitating the operation of the torsion bar.

The suspension system of the track vehicle is designed to transfer the weight of the track vehicle through the track rollers and the track on the ground, to mitigate shocks and impacts acting on the track vehicle hull, and to quickly stop the hull oscillations. The quality of the suspension system to a large extent determines the average speeds of track vehicles on the ground, accuracy of fire on the move, crew performance, reliability and durability of the equipment of the track vehicle. 


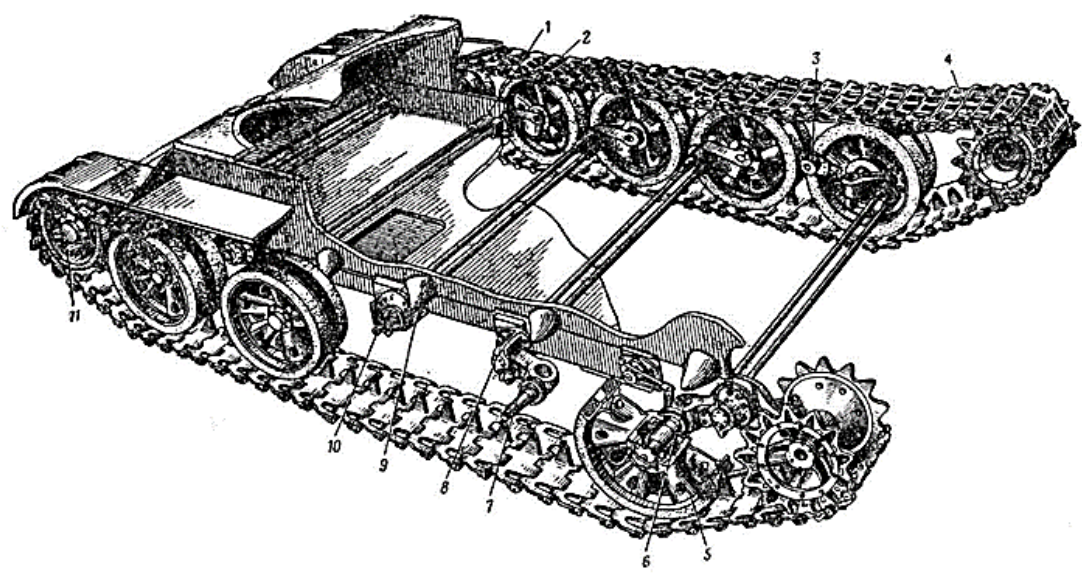

1- Swing arm bracket;

2- Track;

3- Shock absorber;

4- Drive sprocket;

5- Road wheel;

6- Rubber pad;

7- Swing arm;

8- Swing arm support;

9- Bumper;

10- Torsion bar;

11- Idle wheel.

Figure 2. Track vehicle running gear [10].

The track vehicle suspension system includes five suspension assemblies, each of which consists of a road arm, a torsion bar and a bumper. There are hydraulic absorbers on the first and fifth suspension assemblies. As a spring is used a torsion bar with a diameter of $52 \mathrm{~mm}$, made of 45ChNMFA steel, processed by grinding and shot peening, followed by prestressing. Chemical composition and mechanical properties of the torsion bar material are shown in the Table 2.

Table 2. Chemical composition and mechanical properties of the torsion bar material [10].

\begin{tabular}{cccccc}
\hline $\mathbf{C}$ & $\mathbf{S}$ & $\mathbf{P}$ & $\mathbf{M n}$ & $\mathbf{C r}$ & $\mathbf{W}$ \\
\hline $0.42-0.50$ & $\leq 0.025$ & $\leq 0.025$ & $0.50-0.80$ & $0.80-1.10$ & $\leq 0.20$ \\
\hline $\mathbf{V}$ & $\mathbf{T i}$ & $\mathbf{S i}$ & $\mathbf{N i}$ & $\mathbf{M o}$ & $\mathbf{C u}$ \\
\hline $0.10-0.18$ & $\leq 0.030$ & $0.17-0.37$ & $1.30-1.80$ & $0.20-0.30$ & $\leq 0.30$ \\
\hline UTS (MPa) & Yield (MPa) & $\begin{array}{c}\text { Torsion limit } \\
(\mathbf{M P a})\end{array}$ & $\begin{array}{c}\text { Shear modulus } \\
(\mathbf{G P a})\end{array}$ & $\begin{array}{c}\text { Young's modulus } \\
(\mathbf{G P a})\end{array}$ & Hardness HB \\
\hline 1384 & 1080 & 800 & 80 & 205 & 269 \\
\hline
\end{tabular}

\section{4-2- Test Rig Construction}

The test rig was built to test three torsion bars at three different twisting angles to determine the number of cycles to failure at every stress level. A complete test rig equipped with a complete measuring system has been designed and manufactured to measure the moment acting on torsion bar and the corresponding twist angle. The measured parameters are used to evaluate vehicle suspension characteristics. A lifetime of a torsion bar is predicted using the accelerated test in a fatigue test bench. Torsion bars involve repeated torsional loading. Torsional fatigue tests are performed on an axial-type machine. A typical torsional fatigue testing machine is shown in Figure 3.

The test rig consists of a hydraulic power unit, hydraulic cylinder - actuator, and a controller. MTS SilentFlo ${ }^{\mathrm{TM}}$ Hydraulic Power Unit 505.30 delivers superior performance in servo hydraulic testing applications. The MTS Model 493.02 FlexTest SE Controller made by MTS is a fully digital Proportional, Integral, Derivative, Feedforward (PIDF) servo controller. It provides complete control of one servo hydraulic channel or station in an MTS test system.

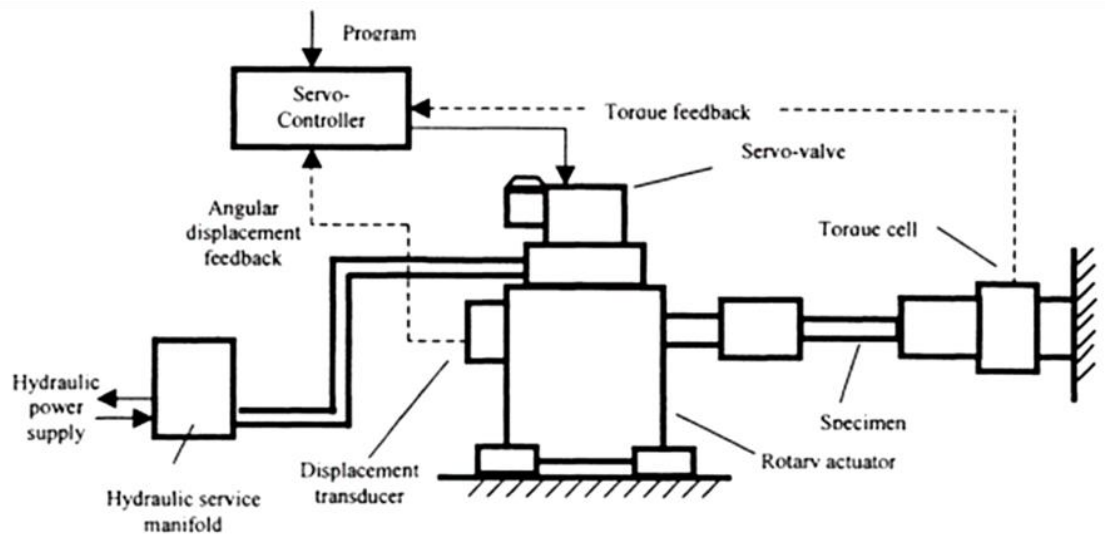

Figure 3. Hydraulic torsional fatigue system [11, 12]. 
The swing angle of the road arm is measured by the Gyro Enhanced Inclinometer FAS-G of the MicroStrain, Inc. The FAS-G not only has the ability to measure static angles, but also dynamic, fast angular movements. Through the use of the two accelerometers and one piezo-ceramic gyro coupled with the requisite digital filtering and embedded software tracking algorithms, FAS-G provides dynamic response while maintaining the DC (static) measurement accuracy. As a result, during rapid angular movements, both the static and the dynamic components of the movement can be measured. The accuracy of the sensor is \pm 1 degree.
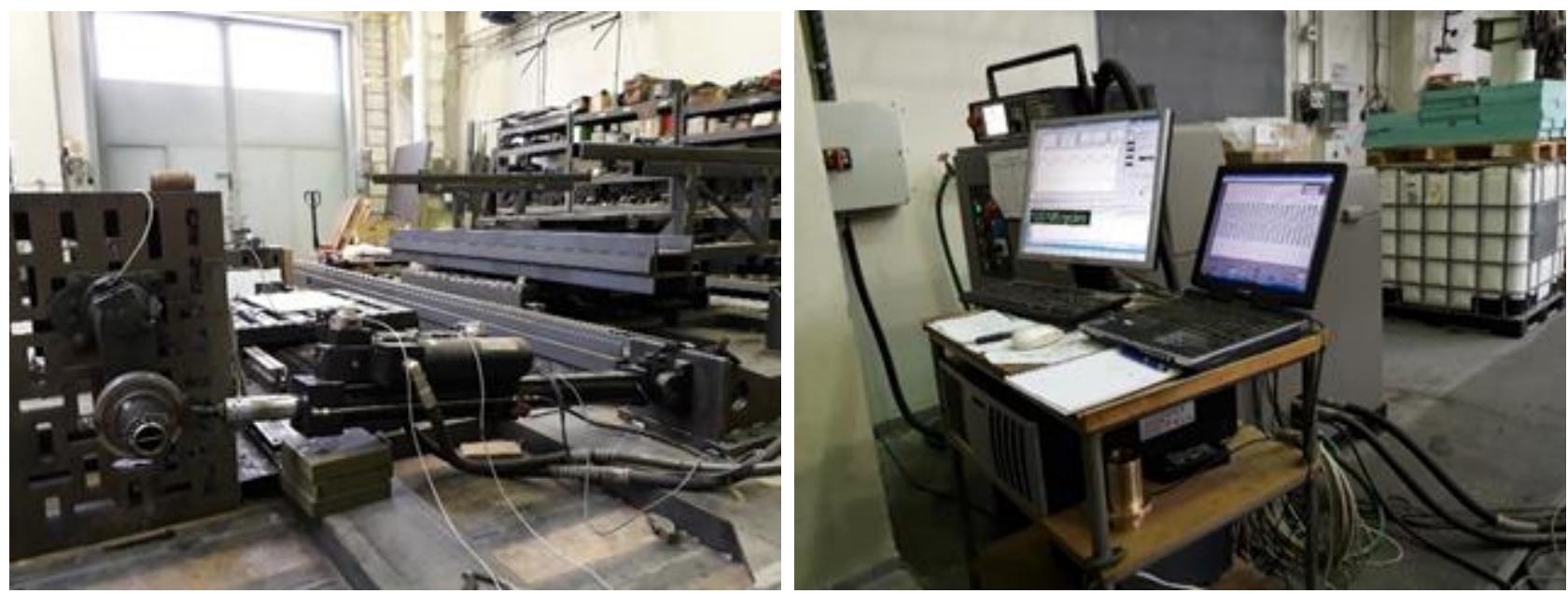

Figure 4. Experimental test rig.

A torsion bar was mounted on the test rig, one end was fixed, and the other end was connected with a swing arm, which rotated about the longitudinal axis of the torsion bar. The first torsion bar was twisted from $15^{\circ}$ to $25^{\circ}$ such as it operated in real life. Note that the static twist angle of the torsion bar is $14.8^{\circ}$. The second torsion bar was twisted from $15^{\circ}$ to $30^{\circ}$. The range of the third torsion bar was from $15^{\circ}$ to $40^{\circ}$. The first and the second torsion bars were swung with frequency $1.5 \mathrm{~Hz}$ while the third torsion bar was with frequency $1 \mathrm{~Hz}$. Every torsion bar went through more than 360000 cycles and no failure was observed. The experimental results are obtained after measuring the applied torque on the torsion bar and the corresponding twist angle.

Due to the limitation of the hydraulic power unit, the test rig was unable to work with the twisting angles of the torsion bar exceeding than $40^{\circ}$.

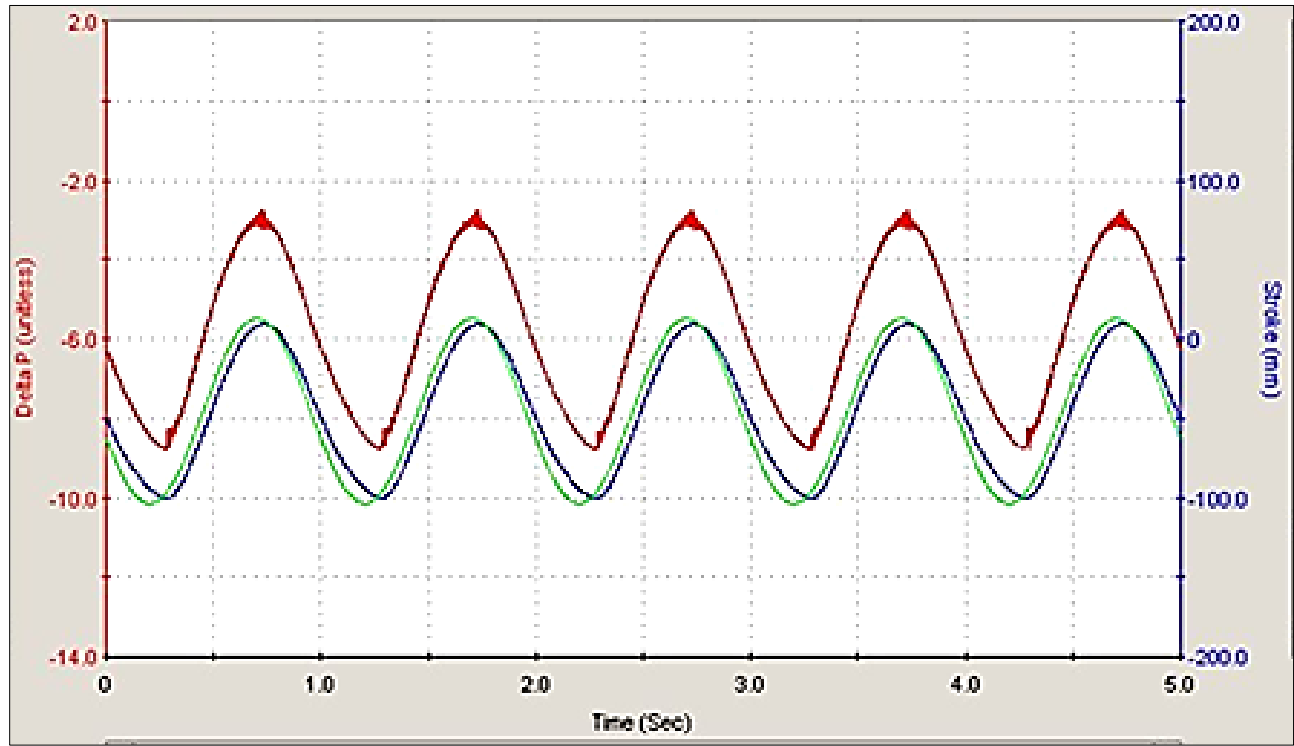

Figure 5. Experiment results rig.

The experimental results were obtained after measuring the applied torque on the torsion bar and the corresponding twist angle. The twisting angle was measured by means of the gyro inclinometer FAS-G while the applied torque was measured by a system of pressure gauges as shown in Figure 5. The summary of the twist angle and the corresponding torque of the torsion bar measured was shown in the Table 3. 
Table 3. Twist angle and torque of torsion bar.

\begin{tabular}{cccccc}
\hline Twist angle, $\left({ }^{\circ}\right)$ & 15 & 25 & 30 & 40 & 53 \\
Twisting moment, $(\mathbf{k N m})$ & 7.7 & 13.0 & 15.5 & 20.6 & - \\
\hline
\end{tabular}

\section{5- Model of Torsion Bar Suspension}

A torsion bar suspension, also known as a torsion spring suspension is a general term for any vehicle suspension that uses a torsion bar as its main weight bearing spring. One end of a long metal shaft is attached firmly to the vehicle body, the opposite end terminates in a road wheel arm, mounted perpendicular to the torsion bar. Vertical motion of the wheel causes the torsion bar to twist around its axis and is resisted by the bar's torsional resistance. The effective spring rate of the bar is determined by its length, diameter, and material.

The advantages of the torsion bar are the possibility to adjust the height of the vehicle, unobtrusiveness compared to coil springs and low cost in design and manufacture. Conversely, it also contains some disadvantages: it is heavy, it provides an inferior ride and handling characteristics and it is difficult to control non-linear spring rates. While tracked vehicle is traveling on road, it is subject to excitation from the road. Vertical motion of the road wheel causes the torsion bar to twist around its axis and is resisted by the bar's torsional resistance. The resistance of the torsion bar to twisting has the same effect as the coil spring used in conventional suspension systems.

From the model of torsion bar suspension as shown in Figure 6, the vertical force exerted on the road wheel and transmitted through the axle arm to the torsion bar is determined by the formula [10]:

$P_{W}=\frac{G J}{L} \frac{\beta}{R \cos \left(\beta_{0}-\beta\right)}$

Where $P_{w}$ - vertical force transmitted from road wheel to the hull; $G$ - shear modulus of torsion bar material; $J$ - polar second moment of torsion bar cross-section; $d$ - diameter of torsion bar; $\beta$ - twist angle of torsion bar; $\beta_{0}-$ setting angle of torsion bar; $L$ - active length of torsion bar.

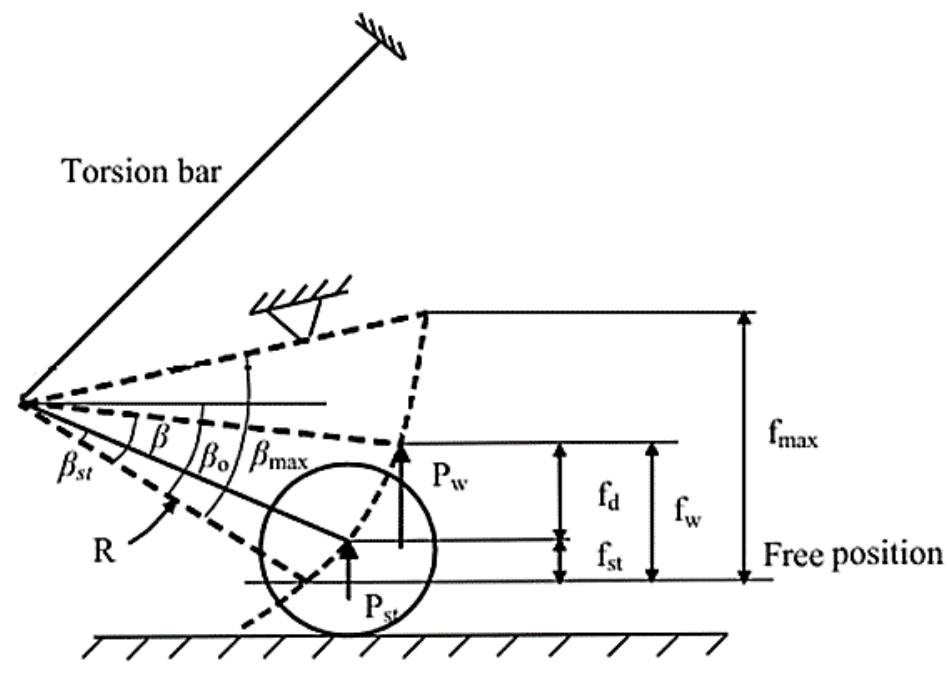

Figure 6. Individual torsion bar suspension $[10,11]$.

This force will cause a torsional moment acting on the torsion bar. The moment is correlated to the resulted twist angle $\beta$ by the following relationship [10].

$T=P_{W} R \cos \left(\beta_{0}-\beta\right)$

Where $T$ - twisting moment on torsion bar; $R$ - radius of road wheel arm.

Therefore:

$T=\frac{G J}{L} \beta=C \beta$

Where $C$ - torsional stiffness of the torsion bar [10].

When a shaft is subjected to a torque or twisting, a shearing stress is produced in the shaft. The shear stress varies from zero in the axis to a maximum at the outside surface of the shaft. Shear stress $\tau$ in the torsion bar is given by:

$\tau=\frac{T}{J} r=\frac{G r}{L} \beta$ 
The torsional stiffness of a uniform rod is dependent on the cross-section diameter, the length, and the shear modulus of elasticity of the rod. Hence, the torsion bar spring rate can be varied by appropriately changing any of the previous parameters [13]. In order to restrict the vertical travel of road wheel as well as safeguard the torsion bar, bumpers are welded on the track vehicle hull. Therefore, the twist angle of the torsion bar reaches the maximum value $\beta_{\max }$ when the swing arm touches the bumper. This angle is smaller than the one corresponding to the shear yield strength of the bar. On the track vehicle, this restrict angle accounts for $53^{\circ}$.

The static angle of the torsion bar $\beta_{s t}$ is approximately $14.8^{\circ}$. The static and maximum twist angles of the torsion bar can be calculated from the schema of individual suspension or measured provided that the setting angle is $\beta_{0}$. The specifications of the individual torsion suspension are shown in the Table 4 and in Table 5 are shown values of twisting moment and shear stress at twist angle.

Table 4. Specifications of torsion bar suspension.

\begin{tabular}{cc}
\hline Parameters & Values \\
\hline Torsion bar diameter, $(\mathrm{mm})$ & 52 \\
Torsion bar active length, $(\mathrm{mm})$ & 1960 \\
Radius of road arm (swing arm), (mm) & 250 \\
\hline
\end{tabular}

Table 5. Values of twisting moment and shear stress at twist angle.

\begin{tabular}{|c|c|c|c|c|c|}
\hline & \multicolumn{2}{|c|}{ Twist angle } & \multirow{2}{*}{$\begin{array}{c}\text { Torque } \\
(\mathrm{kNm})\end{array}$} & \multirow{2}{*}{$\begin{array}{c}\text { Shear stress } \\
(\mathrm{MPa})\end{array}$} & \multirow{2}{*}{$\begin{array}{c}\text { Equiv. stress } \\
\text { (MPa) }\end{array}$} \\
\hline & angular $\left(^{\circ}\right)$ & $(\mathrm{rad})$ & & & \\
\hline Setting position & 0 & 0 & 0 & 255 & \\
\hline \multirow[t]{6}{*}{ Static angle } & 14.8 & 0.258 & 7.6 & 276 & 21 \\
\hline & 25 & 0.436 & 12.9 & 466 & 211 \\
\hline & 30 & 0.524 & 15.4 & 559 & 304 \\
\hline & 40 & 0.698 & 20.6 & 745 & 490 \\
\hline & 45 & 0.785 & 23.1 & 838 & 583 \\
\hline & 50 & 0.873 & 25.7 & 931 & 676 \\
\hline Strict angle & 53 & 0.934 & 27.5 & 996 & 741 \\
\hline
\end{tabular}

\section{6- Test Rig Simulation}

In this article, the model of the test rig was built and tested using MSC Adams software. The virtual model allows simulating the torsion bar at the large twist angles that the test trig cannot. In this model, the torsion bar was represented by a 3D beam comprised of a single FE part of twelve nodes. One end is constrained with the ground and the other end is constrained with the swing arm (Figure 7).

The FE part is a wholly Adams-native modelling object with inertia properties and is accurate for very large deformation cases of beam-like structures. The FE part differs from the linear flexible body option within Adams Flex in two significant ways. Firstly, it has the ability to accurately represent large deformations which the linear modes approach cannot. Secondly, its modelling does not require an FEA produced file like the modal neutral file. The FE Part also differs from the beam force element in that it possesses inertia properties [14, 19, 20]. For the test rig model and through the experiment, the following data were applied: torsion bar diameter - $52 \mathrm{~mm}$, torsion bar active length $1960 \mathrm{~mm}$, shear modulus of torsion bar material $-80.23 \mathrm{kN} / \mathrm{mm}^{2}$.

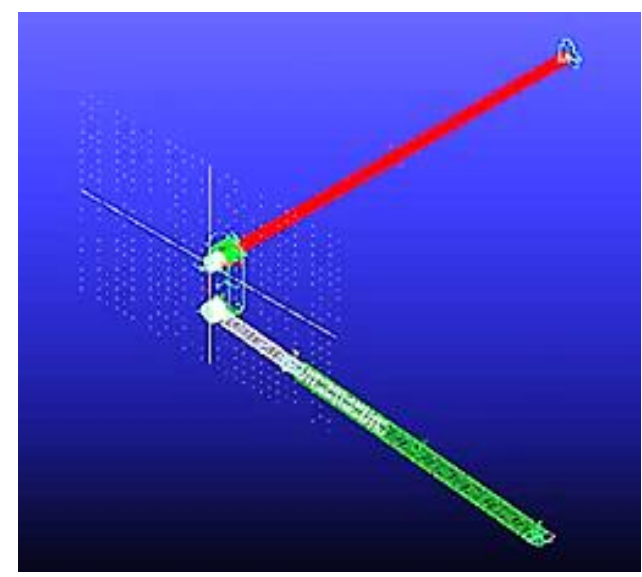

Figure 7. Model of experiment setup rig. 
Modelling and simulation are two slightly different processes but usually carried out together. They are used to make models based on a real object and allow us to experiment with these models [15].

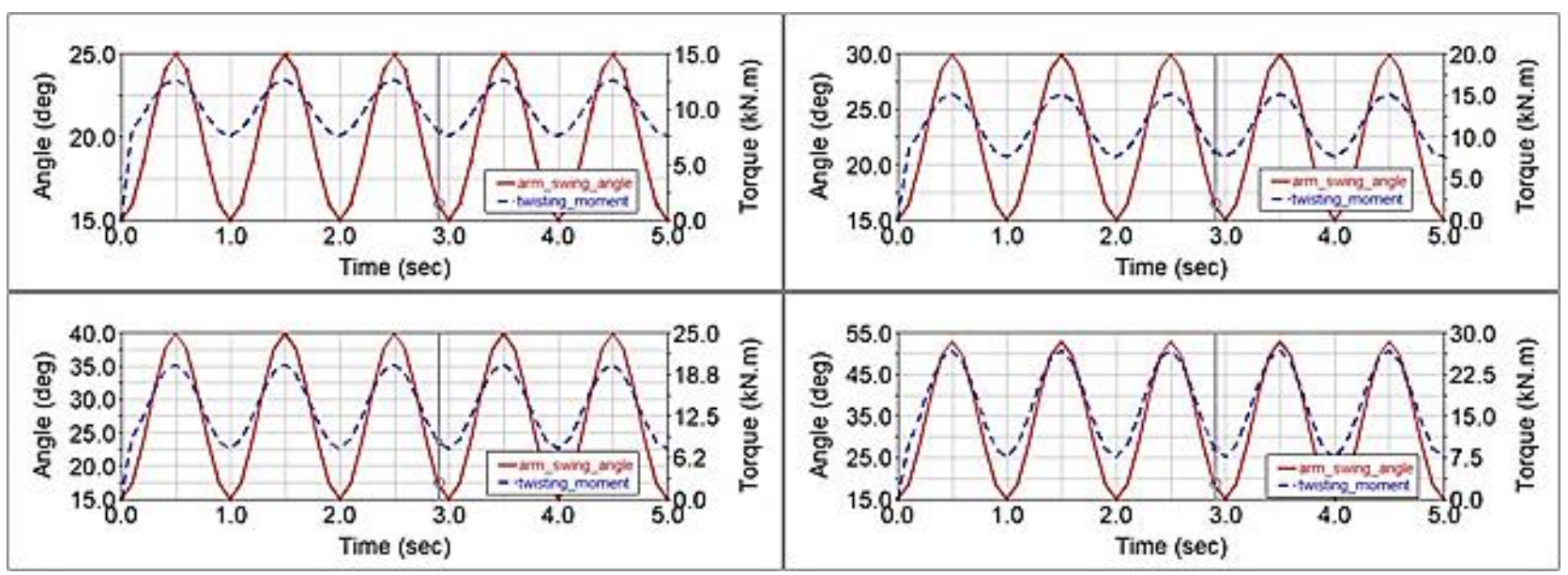

Figure 8. Simulation results for twisting moment and swing angle of road arm [16].

Created model make possible to simulate the dynamic analysis, obtained the twist angle and the torque of torsion bar and compare to the real test results. The comparison is shown in the Table 6.

Table 6. Comparison of experimental and simulation results [11].

\begin{tabular}{ccccccc}
\hline & Twist angle in $\left({ }^{\circ}\right)$ & 15 & 25 & 30 & 40 & 53 \\
\hline \multirow{2}{*}{ Twisting } & Experiment $(\mathbf{k N m})$ & 7.7 & 13.0 & 15.5 & 20.6 & - \\
moment & Simulation $(\mathbf{k N m})$ & 7.6 & 12.7 & 15.2 & 20.3 & 26.9 \\
\hline
\end{tabular}

It is clear from the table that the results of physical tests and simulation have an insignificant difference.

\section{7- Using Monte Carlo Method for Accelerated Life Test}

Monte Carlo simulation provides a powerful, insightful tool for planning experiments. For a specified model and planning values for the model parameters, it is possible to use a computer to simulate ALT experiments to see the kind of data that will be obtained and to visualize the variability from trial to trial. Such simulations provide an assessment of sampling uncertainty that will result from using a limited number of test specimens.

The basic idea of this method is very simple; we want to determine the mean value of a quantity that is the result of a random process. A computer model of this process is created and after a sufficient number of simulations, the data can be processed by classical statistical methods, for example to determine the mean and determinant deviation. The MC method includes:

- Creation of a real system model, with the same probability characteristics as in the real system (influence of chance - random numbers),

- The model must include all relevant facts that significantly affect the real system,

- Experimenting with the model, multiple exploration of the model behaviour

- With fixed time step - we monitor the behaviour of the system after certain constant time intervals to find out if changes have occurred,

- With variable time step - we generate an interval during which no changes occur in the system.

For a specified model, planning values for the model parameters, and test plan, the large-sample approximations methods allow one to compute the large-sample approximate variance-covariance matrix of the maximum likelihood estimators of the model parameters $\theta$. Using this matrix, it is easy to compute large-sample approximate standard errors of maximum likelihood estimates and these easy-to-compute quantities are useful for comparing different test plans. 
In order to get failure data at the specified test stress levels, the Monte Carlo utility of ALTA (Accelerated Life Testing Analysis) software is exploited. It uses Monte Carlo simulation to generate a single data set containing values that are distributed according to a specified life distribution or model, namely Weibull distribution. With Monte Carlo utility, the number of points in a single data set can be chosen freely. It needs to specify the shape $\beta$ and scale parameters $\eta$ for each test level for the software to produce random points. The shape parameter of a product for specific failure mechanism is independent of stress level and there $\beta=2.03$. The scale parameter varies with stress and can be determined with the help of the S-N curve of the torsion bar material [17], showed in Figure 9.

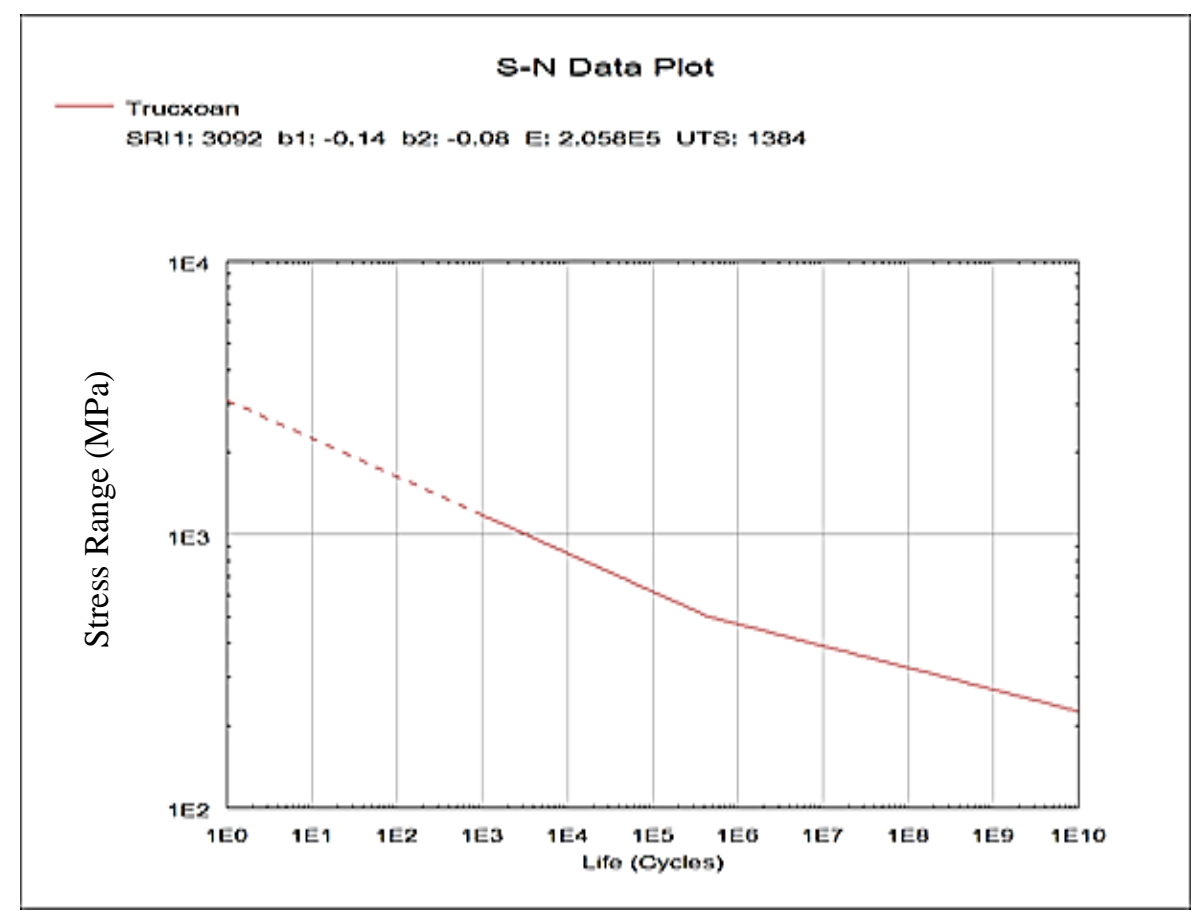

Figure 9. Stress - life curve of torsion bar [17].

From the S-N curve, the number of cycles to failure was determined for each stress level. We used the Weibull distribution, which is obtained by considering the probability density function (pdf) for the 2-parameter Weibull distribution. It is given by Vassiliou and Mettas (2001) [18].

$f(t)=\frac{\beta}{\eta}\left(\frac{t}{\eta}\right)^{\beta-1} e^{-\left(\frac{t}{\eta}\right)^{\beta}}$

The mean life mean time to failure (MTTF) of the 3-parametres Weibull probability density function is given by Vassiliou and Mettas (2001) [18].

$M T T F=\gamma+\eta \Gamma\left(\frac{1}{\beta}+1\right)$

Where $\Gamma\left(\frac{1}{\beta}+1\right)$ is gamma function evaluated at the value of $\left(\frac{1}{\beta}+1\right)$.

This number can be considered the mean life of the torsion bar and the scale parameter is found by the Equation 9 . The values of scale parameter for each stress level are shown in the Table 7.

Table 7. Values of scale parameter for each stress level.

\begin{tabular}{cccc}
\hline & High level & Medium level & Low level \\
\hline Shear stress, MPa & 676 & 583 & 490 \\
Twist angle, ${ }^{0}$ & 50 & 45 & 40 \\
Shape parameter & & 2.03 & \\
Scale parameter & 58742 & 169072 & 585008 \\
\hline
\end{tabular}

Providing the shape, scale parameters and the number of points for each stress level, ALTA generated the failure data set, which is presented in the Table 8 . 
We calculated the parameters of the IPL-Weibull model by the Equations 10 to 13 using the maximum likelihood estimation (MLE) method for the above generated data set in ALTA software. The obtained parameter estimates of the model are summarized in the Table 9.

The IPL-Weibull model can be derived by setting $\eta=L(V)$ in the Weibull probability density function, yielding the following IPL-Weibull probability density function:

$f(t, V)=\beta K V^{m}\left(K V^{m} t\right)^{\beta-1} e^{-\left(K V^{m} t\right)^{\beta}}$

The mean life MTTF of the IPL-Weibull model is given by

$$
M T T F=\frac{1}{K V^{m}} \Gamma\left(\frac{1}{\beta}+1\right)
$$

Where $\Gamma\left(\frac{1}{\beta}+1\right)$ is gamma function evaluated at the value of $\left(\frac{1}{\beta}+1\right)$.

Table 8. Number of cycles to failure at different twist angles.

\begin{tabular}{cc}
\hline Number of cycles to failure (cycles) & Stress level $\left(^{\circ}\right)$ \\
\hline 966451 & 40 \\
1275323 & 40 \\
888890 & 40 \\
635947 & 40 \\
129792 & 40 \\
557513 & 40 \\
512498 & 40 \\
725703 & 40 \\
194244 & 40 \\
279312 & 45 \\
368579 & 45 \\
256896 & 45 \\
183794 & 45 \\
37511 & 45 \\
97044 & 50 \\
128058 & 50 \\
89255 & 50 \\
63857 & 50 \\
13033 & 50 \\
55981 & 50 \\
\hline
\end{tabular}

The IPL-Weibull reliability function is given by

$R(t, V)=e^{-\left(K V^{m} t\right)^{\beta}}$

The IPL-Weibull failure rate function, $\lambda(t)$, is given by

$\lambda(t, V)=\frac{f(t, V)}{R(t, V)}=\beta K V^{m}\left(K V^{m} t\right)^{\beta-1}$

Table 9. Regression parameters for IPL-Weibull model.

\begin{tabular}{ccc}
\hline $\boldsymbol{\beta}$ & $\boldsymbol{K}$ & $\boldsymbol{m}$ \\
\hline 2.008878 & $3.101666 \mathrm{E}-22$ & 9.758032 \\
\hline
\end{tabular}

The parameter $m$ in the inverse power relationship is a measure of the effect of the stress on the life. As the absolute value of $m$ increases, the greater is the effect of the stress. Negative values of $m$ indicate an increasing life with increasing stress. An absolute value of $m$ approaching zero indicates small effect of the stress on the life, with no effect (constant life with stress) when $m=0$.

Next, a likelihood ratio test is performed to assess the assumption of a common shape parameter among the data obtained at the various accelerated stress levels. This assumption underlies the use of any model that includes a life 
distribution with a shape parameter. By confirming this assumption, it is confirmed that units will fail in the same manner across different stress levels.

Then a significance level $=0.1$ is chosen, the ALTA determines whether the estimated shape parameters are statistically the same at a confidence level of 0.9 .

The results of the likelihood ratio test were as follows:

- Significance level $\alpha=0.1$;

- Shape parameter $\beta=2.009$;

- The likelihood ratio test statistic $T=0.025$;

- Chi-squared $\left(\alpha_{\mathrm{j}-1}\right)=4.605$.

Since the value of the likelihood ratio test statistic $T$ is less than the chi-squared value, the shape parameter estimates do not differ statistically at the level $10 \%$. After fitting the model, results of the accelerated test can be extrapolated back to normal-use conditions. Here we were interested in the mean life of the torsion bar at normal use conditions, showed in Figure 10.

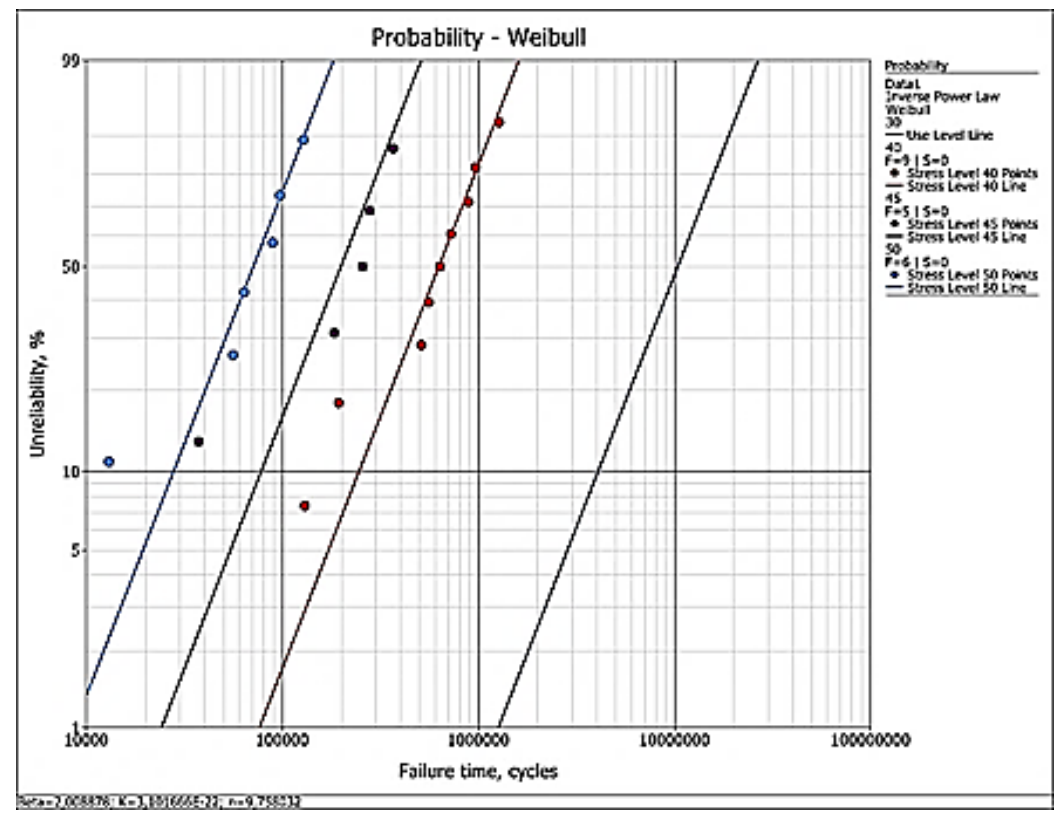

Figure 10. Probability plot rig.

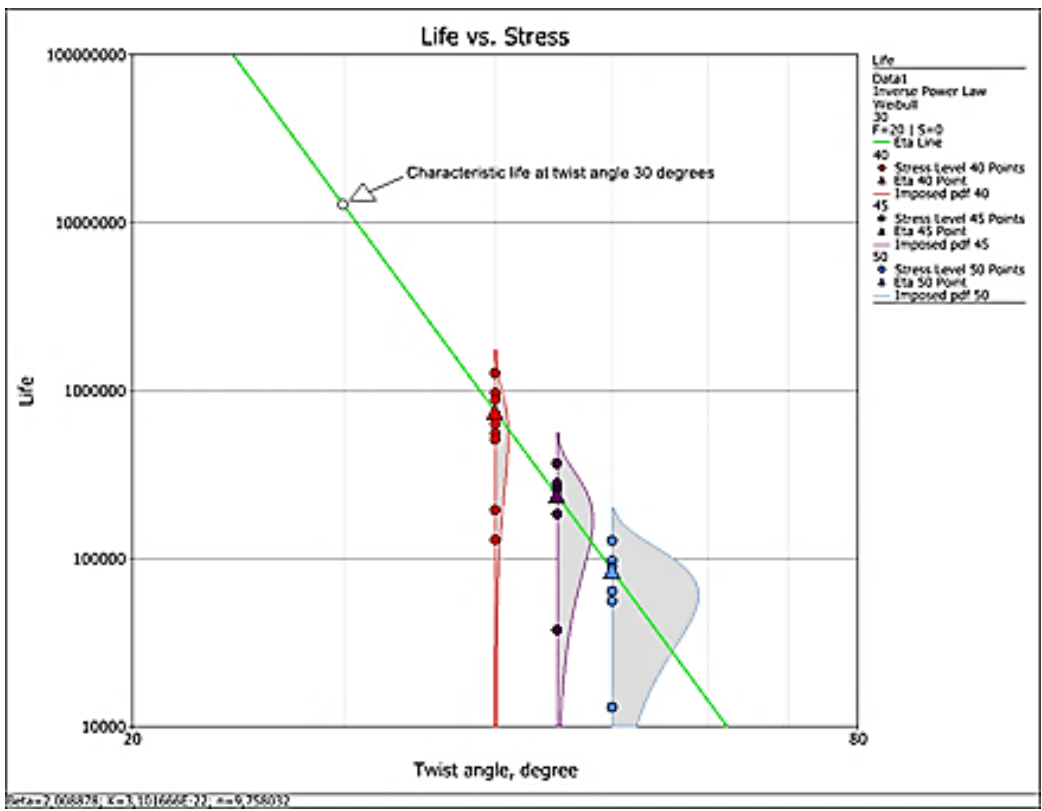

Figure 11. Life vs. stress plot. 
The life vs. stress plot shows in Figure 11 the effect of a stress on the life of the product. Multiple probability density function, each at a different stress level used in the test, is displayed on the plot. The failure times obtained at each stress level are shown at the base of the associated probability density function. Note that the lines and probability density function are mapped to the use stress level, but the failure times are plotted at the tested conditions.

As the Weibull distribution was selected, an eta line was displayed as well. The eta line estimates the time by which $63.2 \%$ of units in the population are expected to fail. The mean life of the torsion bar at normal condition is more than 11 millions cycles. The mean life of the torsion bar at different stress level is in the Table 10.

Table 10. Mean life at different stress level.

\begin{tabular}{ccccc}
\hline Twist angle $\left(^{0}\right)$ & 30 & 40 & 45 & 50 \\
\hline Mean life (cycles) & 11018410 & 665216 & 210773 & 75390 \\
\hline
\end{tabular}

\section{8- Conclusions}

This article deals with the accelerated life test for a torsion bar of the track vehicle. Majority of mechanical damages of structural elements occur due to material fatigue during its normal operation. Fatigue is a major failure mechanism of mechanical parts. At the same time, this type of damage is considered to be the most dangerous one, the crack initiation in structural elements is difficult to predict, and it leads to damage of the elements.

The primary purpose of an ALT is to estimate the life distribution and quantities of interest in a use condition. This estimation involves extrapolation from higher stress levels by using an acceleration model and thus includes the model error and statistical uncertainty. For the torsion bars of tracked combat vehicles with stringent functionality specifications, the reliability is very high. Therefore, their life testing under nominal conditions requires much time and resources. In response to this problem, ALT finds an application to obtain timely information on the reliability of the products.

The following results were obtained:

- The model of the test rig was built and measurement was performed;

- The dynamic simulation was performed;

- The simulation results were compared with experimental results obtained on the experimental test rig. The comparison showed a good agreement between the results, which proved the accordance of the model;

- The proposed model allows saving money and simulating various torsion bar fatigue processes, including lifetime prediction. This simulation allows determining different loads, frequencies and times of the experiment;

- In our realized experiment, we did not detect the failure - lifetime of the torsion bar, because we expected a lower lifetime of the torsion bar of the track vehicle;

- The aim of the proposed model is to predict the lifetime of the torsion bar;

Therefore, we used the Monte Carlo method, which generates data sets for stress level according to the accelerated test plan. The results of data analysis demonstrated the reliability merits of the torsion bar including mean life.

Lifetime prediction can be made for a mechanical component with the help of a damage accumulation hypothesis. The prediction can only be made with a certain probability since the load spectrum, as well as the load capacity expressed in the form of an S-N curve, are random variables. Likewise, the damage accumulation hypotheses have only been proven empirically in materials science. Therefore, a practical lifetime prediction requires a balance between field tests, test stand trials, calculations and evaluation of the data.

\section{9- Acknowledgments}

Presented work has been prepared with the support of the Ministry of Defence of the Czech Republic, Partial Project for Institutional Development and Specific Research, Department of Combat and Special Vehicles, University of Defence, Brno.

\section{0- Conflict of Interest}

The author declares that there is no conflict of interests regarding the publication of this manuscript. In addition, the ethical issues, including plagiarism, informed consent, misconduct, data fabrication and/or falsification, double publication and/or submission, and redundancies have been completely observed by the authors. 


\section{1- References}

[1] Neumann, Vlastimil, Zdenek Krobot, and Tomas Turo. "Stress of a Vehicle Propulsion Mechanism." 2017 International Conference on Military Technologies (ICMT) (May 2017). doi:10.1109/miltechs.2017.7988752.

[2] Mihon, L, N Lontiş, and S Deac. "The Behaviour of a Vehicle's Suspension System on Dynamic Testing Conditions.” IOP Conference Series: Materials Science and Engineering 294 (January 2018): 012083. doi:10.1088/1757-899x/294/1/012083.

[3] Nguyen, Q. H., Furch, J. “Analysis of Stress Distribution of Tracked Vehicle Torsion Bar”. 2018 22nd International conference Transport Means.

[4] IEC 62506:2013. Methods for Product Accelerated Testing.

[5] B. Bertsche. "Reliability in Automotive and Mechanical Engineering - Determination of Component and System Reliability", 2008. doi:10.1007/978-3-540-34282-3.

[6] Meeker, William Q., and Luis A. Escobar. "Statistical methods for reliability data", (July 24, 1998).

[7] W. B. Nelson. “Accelerated Testing - Statistical Models, Test Plans, and Data Analysis”, (September 21, 2004).

[8] G. Yang. "Life Cycle Reliability Engineering”, (February 2, 2007).

[9] Leitner, Bohuš, and Lucia Figuli. "Fatigue Life Prediction of Mechanical Structures under Stochastic Loading." Edited by M. Vasko, M. Handrik, L. Jakubovičová, P. Kopas, M. Blatnická, V. Baniari, O. Štalmach, A. Sapietová, and M. Sága. MATEC Web of Conferences 157 (2018): 02024. doi:10.1051/matecconf/201815702024.

[10] Sergeev, L. V. "Theory of tank. Moscow”, 1973.

[11] Nguyen, Quy Hung, and Jan Furch. "Mathematical Model for Vibration Analysis of Tracked Vehicle." 2019 International Conference on Military Technologies (ICMT) (May 2019). doi:10.1109/miltechs.2019.8870060.

[12] ASM International. Handbook Committee. “ASM Handbook: Mechanical testing and evaluation”, Vol. 8. 2000.

[13] Novotný, Pavel, Jozef Hrabovský, Jaroslav Juračka, Jiří Klíma, and Vladimir Hort. "Effective Thrust Bearing Model for Simulations of Transient Rotor Dynamics.” International Journal of Mechanical Sciences 157-158 (July 2019): $374-383$. doi:10.1016/j.ijmecsci.2019.04.057.

[14] Mechanical dynamics. Building Models in ADAMS/View. Ann Arbor, Michigan, 2000.

[15] Furch, Jan, and Trung Tin Nguyen. "Simulation of Failure in Gearbox Using MSC.Adams." Acta Universitatis Agriculturae et Silviculturae Mendelianae Brunensis 65, no. 2 (2017): 419-428. doi:10.11118/actaun201765020419.

[16] Nguyen, Q. H., Furch, J. “Model for Investigating Characteristics of Tracked Vehicle Torsion Bar”. 2019 23rd International Scientific Conference Transport Means.

[17] Wang, Hongyan, Qiang Rui, and Xiaojun He. "The Prediction Technology Study of Fatigue Life for Key Parts of a Tracked Vehicle's Suspension System." Frontiers of Mechanical Engineering in China 2, no. 1 (March 2007): 68-71. doi:10.1007/s11465-007-0011-0.

[18] Vassiliou, Pantelis, and Adamantios Mettas. "Understanding accelerated life-testing analysis." Annual Reliability and Maintainability symposium, Tutorial Notes. 2001.

[19] Barsoum R. S.; Bethony R. H.; Brockelman H. P.; Hatch H. P.; Hickey J.; Matthews W. T.; Neal D. M. Reliability and Life Prediction Methodology - M60 Torsion Bars. Army Material and Mechanics Research Center. Massachusetts. 1985.

[20] Będkowski, Włodzimierz. "Assessment of the fatigue life of machine components under service loading a review of selected problems." Journal of Theoretical and Applied Mechanics 52, no. 2 (2014): 443-458.

[21] Biroloni A. Reliability Engineering-Theory and Practice. 8th ed. Berlin. Springer. 2017.

[22] Ismail Azianti; Jung Won; Liu Qiang. Accelerated Life Test Design for Tractor Powertrain Front Axle. ICMER 2015. 2016, $74,00020$.

[23] Meeker, William Q., Luis A. Escobar, and Yili Hong. "Using accelerated life tests results to predict product field reliability." Technometrics 51, no. 2 (2009): 146-161.

[24] Zaharia S. M.; Martinescu I. M.; Morariu C. O. Lifetime Prediction using Accelerated Test Data of the Specimens from Mechanical Element. Maintenance and Reliability. 2012, 14(2): 99-106. 